\title{
SYN1 Gene Mutation in a Child with Focal Epilepsy and Reflex Bathing Seizures
}

\author{
Deepa Sirsi $^{1}$ Dallas Armstrong ${ }^{1}$ Jorge Muñoz-Bibiloni ${ }^{1,2}$ Bridget Redondo ${ }^{3}$ Jason Y. Park ${ }^{4}$
}

${ }^{1}$ Department of Pediatric Neurology, University of Texas

Southwestern Medical Center, Dallas, Texas, United States

2 San Jorge Children's Hospital and Hospital San Francisco, San Juan, Puerto Rico, United States

${ }^{3}$ Department of Pediatric Psychology, Children's Medical Center, Dallas, Texas, United States

${ }^{4}$ Department of Pathology and the Eugene McDermott Center for Human Growth and Development, University of Texas Southwestern Medical Center, Dallas, Texas, United States

J Pediatr Epilepsy 2017;6:119-124.

\author{
Address for correspondence Deepa Sirsi, MD, Department of \\ Pediatric Neurology, University of Texas Southwestern Medical \\ Center, 5323 Harry Hines Boulevard, Dallas, TX 75390, United States \\ (e-mail: deepa.sirsi@UTSouthwestern.edu).
}

\begin{abstract}
Keywords

- reflex epilepsy

- SYN1 gene mutation

- bathing epilepsy

- vagal nerve stimulator

- autism

Reflex seizures are evoked by a specific afferent stimulus or activity. The pathophysiology of reflex seizures is thought to be hyperexcitability of cortical or subcortical neuronal areas that respond to a physiologic stimulus in an exaggerated manner. This hyperexcitability could occur due to underlying lesions or genetic abnormalities. We present a 7-year-old Latino boy with epilepsy, autism spectrum disorder, developmental impairment, and reflex seizures triggered by water and bathing. His seizures were refractory to antiseizure medications. After multiple trials of medications, a vagal nerve stimulator was placed and desensitization therapy initiated. An epilepsy gene panel test was obtained, which showed a mutation in the SYN1 gene (c.1264C > T het, p. $\operatorname{Arg} 422^{*}$ ). There is only one other publication of a family with SYN1 gene mutation and reflex bathing seizures. Identification of this correlation may be helpful in recognition and treatment of other patients and families with this rare presentation.
\end{abstract}

\section{Introduction}

According to the most recent definition by the International League Against Epilepsy, a reflex seizure is "objectively and consistently demonstrated to be evoked by a specific afferent stimulus or by activity of the patient." The afferent stimuli or activity can be either elementary or elaborate., Reflex seizures, when always provoked by one specific stimulus, are traditionally called reflex epilepsy, but they can occur along with spontaneous seizures. ${ }^{3,4}$ Their pathophysiology is thought to be hyperexcitability of cortical or subcortical neuronal areas that respond to a physiologic stimulus in an exaggerated manner. This hyperexcitability could occur due to underlying lesions or genetic abnormalities. $^{5}$

received

June 28, 2016

accepted after revision

August 25, 2016

published online

March 7, 2017
Photosensitive reflex seizures are the most common type of reflex seizures. ${ }^{6}$ Other triggers such as music, reading, eating, and movement are less common, especially in children. Hot water epilepsy is common in children in certain parts of the world, and genetic loci at chromosomes 10q21.3-q22.3 and 4q24-q28 have been identified through linkage analysis in families, but apart from this reflex bathing seizures are not common. ${ }^{6,7}$ Reflex bathing seizures are not related to hot water, and bathing seizures may invoke a pathophysiology different from that of elevated temperature with genetic susceptibility in hot water seizures. $^{7}$

Reflex bathing seizures, epilepsy, and autism spectrum disorder have been linked to synapsin 1 (SYN1) gene mutation in one large French-Canadian family. ${ }^{8}$ We report a Latino
Copyright $\odot 2017$ by Georg Thieme Verlag KG, Stuttgart . New York
DOI https://doi.org/ 10.1055/s-0037-1599193. ISSN 2146-457X. 
patient with SYN1 mutation, epilepsy, cognitive impairment, autism spectrum disorder, and reflex bathing seizures to add to the knowledge about the clinical spectrum of this mutation.

\section{Patient Description}

A 7-year-old right-handed Latino boy presented with seizures since 3 years of age. Birth history, early development, and neurologic examination were normal. The patient had significant family history of epilepsy. Two maternal cousins had epilepsy. One of the maternal aunt's son's epilepsy was controlled with carbamazepine. Mother's other sister's son was weaned off medications at age 14 without seizure recurrence. He also had a maternal uncle with epilepsy taking unspecified medications, and his maternal grandmother had multiple seizures occurring only during pregnancy. There was insufficient information about seizure semiology in family members, but bathing and water triggers were not reported.

His seizure frequency was one to two per month, and in early childhood, some of his seizures were triggered by defecation. His epilepsy became medically intractable at $4 \frac{1}{2}$ years of age. After age 5 , seizures tended to occur during or soon after showering, or when water was poured over his face and shoulders. Semiology consisted of looking nervous, lip smacking with balling of fists, perioral cyanosis, and was followed by postictal sleep for 2 to 3 hours. He stopped taking baths, and his mother was washing him with a sponge. Three tesla magnetic resonance imaging (MRI) of the brain was normal. Electroencephalogram (EEG) at age 5 years showed left temporal spikes.

Two seizures were recorded during a presurgical video EEG monitoring. The first seizure was triggered by bathing and the second by washing his face in water at body temperature. The semiology consisted of staring and unre- sponsiveness, bilateral arm and leg extension, and stiffening during the first seizure. There were staring, unresponsiveness, followed by right arm abduction at the shoulder and flexion at the elbow and subsequent bilateral arm flexion and leg extension during the second seizure. The clinical semiology was not lateralizing or localizing. The electrographic seizure onset was not well lateralized with the first seizure (-Fig. 1), but it evolved over the left temporal region (-Fig. 2). During the second seizure, ictal evolution was in the right frontotemporal region (-Fig. 3). Interictal EEG was significant for generalized-appearing spikes (-Fig. 4). Ictal and interictal single photon emission computed tomography (SPECT) scans showed symmetric uptake throughout the brain. Neuropsychological testing was not localizing or lateralizing, but the patient's deficits across multiple domains suggested diffuse cerebral dysfunction. A gene panel test of 40 genes focused on childhood-onset epilepsy was performed (GeneDx Childhood Epilepsy Panel, version 2013, Gaithersburg, Maryland, United States). This test for 40 genes examines both nucleotide variants by DNA sequencing and gene duplication/deletion by oligonucleotide microarray. This test identified two novel variants in the genes: SYN1 (c.1264C > T hem, p.Arg422*) (-Fig. 5) and POLG (c.2749G $>$ A het, p.Gly917Arg). Although both gene variants have not been previously described, the SYN1 variant is a stop mutation predicted to result in loss in protein function. In addition, mutations in SYN1 are X-linked conditions whereas POLG-related disorders with epilepsy are typically autosomal recessive conditions; this patient only had the single POLG variant. Familial genetic testing to examine the inheritance pattern of the variants was precluded by financial reasons.

His antiseizure medication (ASM) trials included levetiracetam, zonisamide, oxcarbazepine, and his current regimen of lamotrigine and carbamazepine. He developed a fear of running water in response to his seizures and was referred to

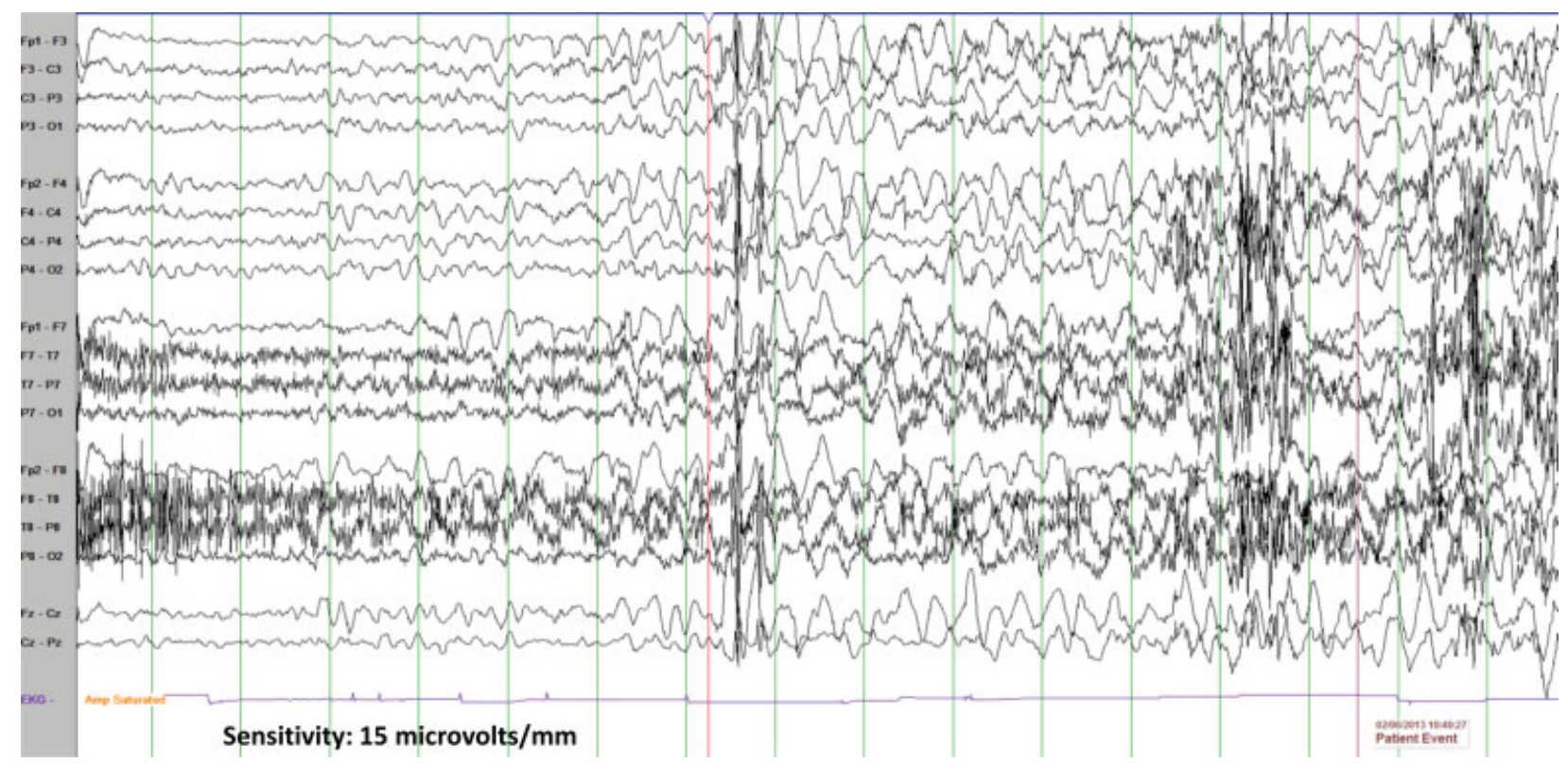

Fig. 1 Ictal EEG, seizure 1 onset. Seizure triggered by bathing. Generalized spikes and diffuse rhythmic delta at seizure onset. 


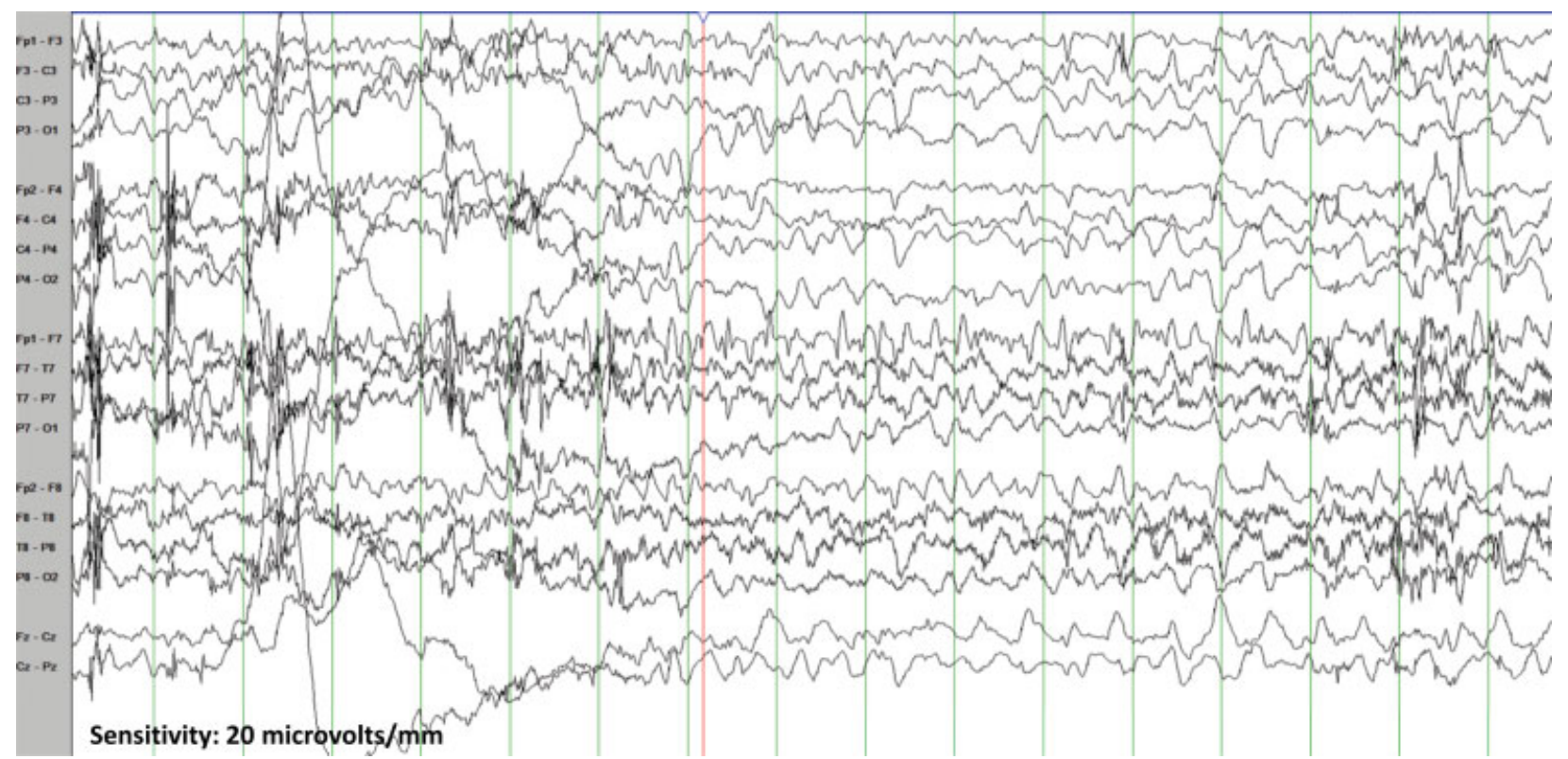

Fig. 2 Ictal EEG, seizure 1 progression. Seizure triggered by bathing. Left temporal rhythmic spikes within 15 seconds of seizure onset.

psychology for desensitization therapy. With therapy, he had improvement in coping when exposed to water. He was able to pour water over his face and take showers regularly with rare bathing-induced breakthrough seizures. However, he continued to have spontaneous nocturnal seizures for which clonazepam was added, which helped reduce these seizures. Because of medically intractable seizures, a vagal nerve stimulator (VNS) was placed at age 10 years. At the last follow-up 1 year after VNS placement, seizures were reduced by 40 to $50 \%$ with less frequent and shorter unprovoked seizures. His reflex bathing seizures were not significantly improved with the addition of VNS. His VNS settings were at output current $1.75 \mathrm{~mA}(\mathrm{~mA})$ signal on time 30 seconds, off time 5 minutes, magnet current $2 \mathrm{~mA}$.

\section{Discussion}

Nguyen et al described a cohort of a large French-Canadian family with reflex bathing epilepsy and SYN1 mutation (c.1663C > T, p.Gln555*; also reported as Q555X). ${ }^{8}$ Mutations in SYN1 have also been identified in families with multiple males having autism, neurodevelopmental

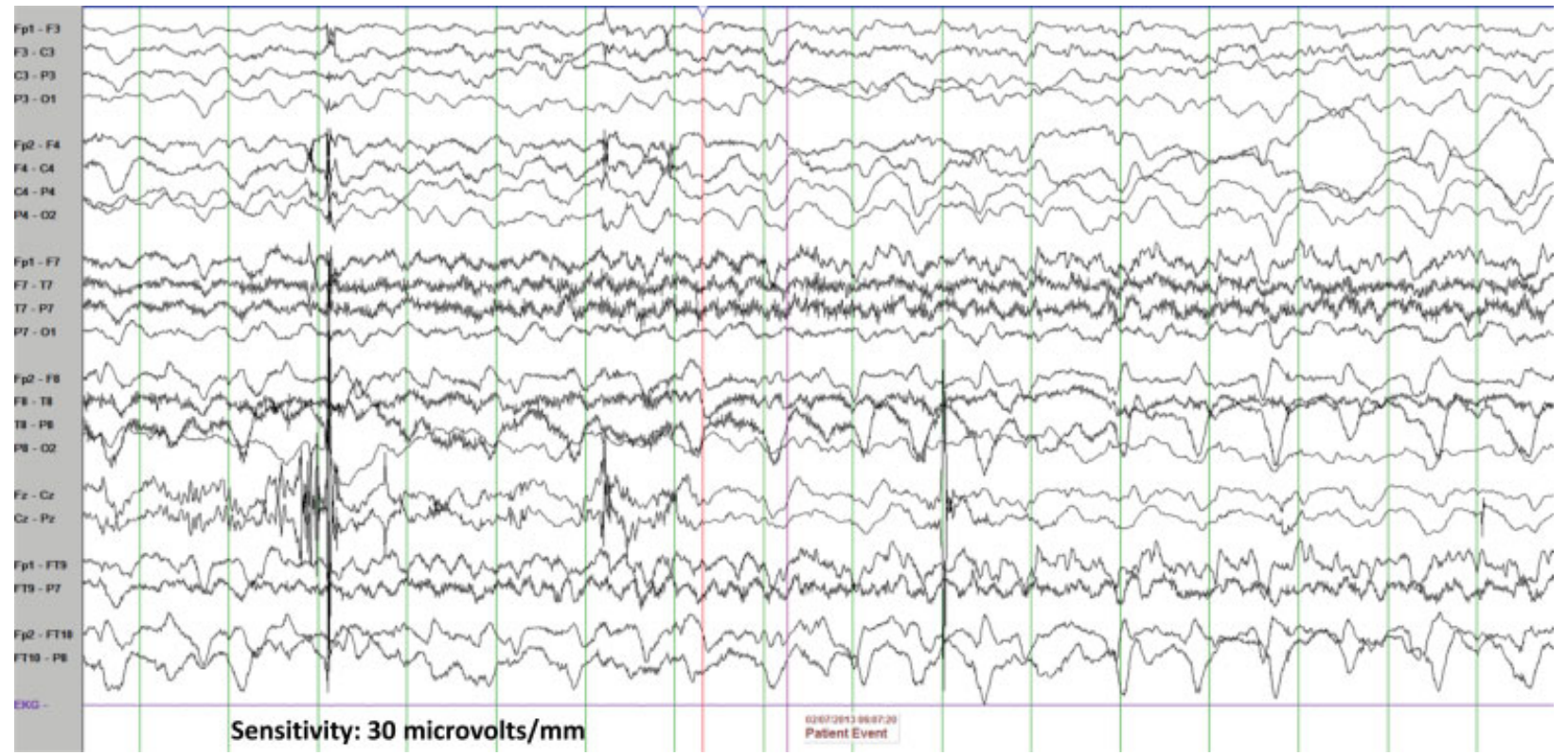

Fig. 3 Ictal EEG, seizure 2 onset. Seizure triggered by washing face. Right frontotemporal rhythmic delta at seizure onset. 


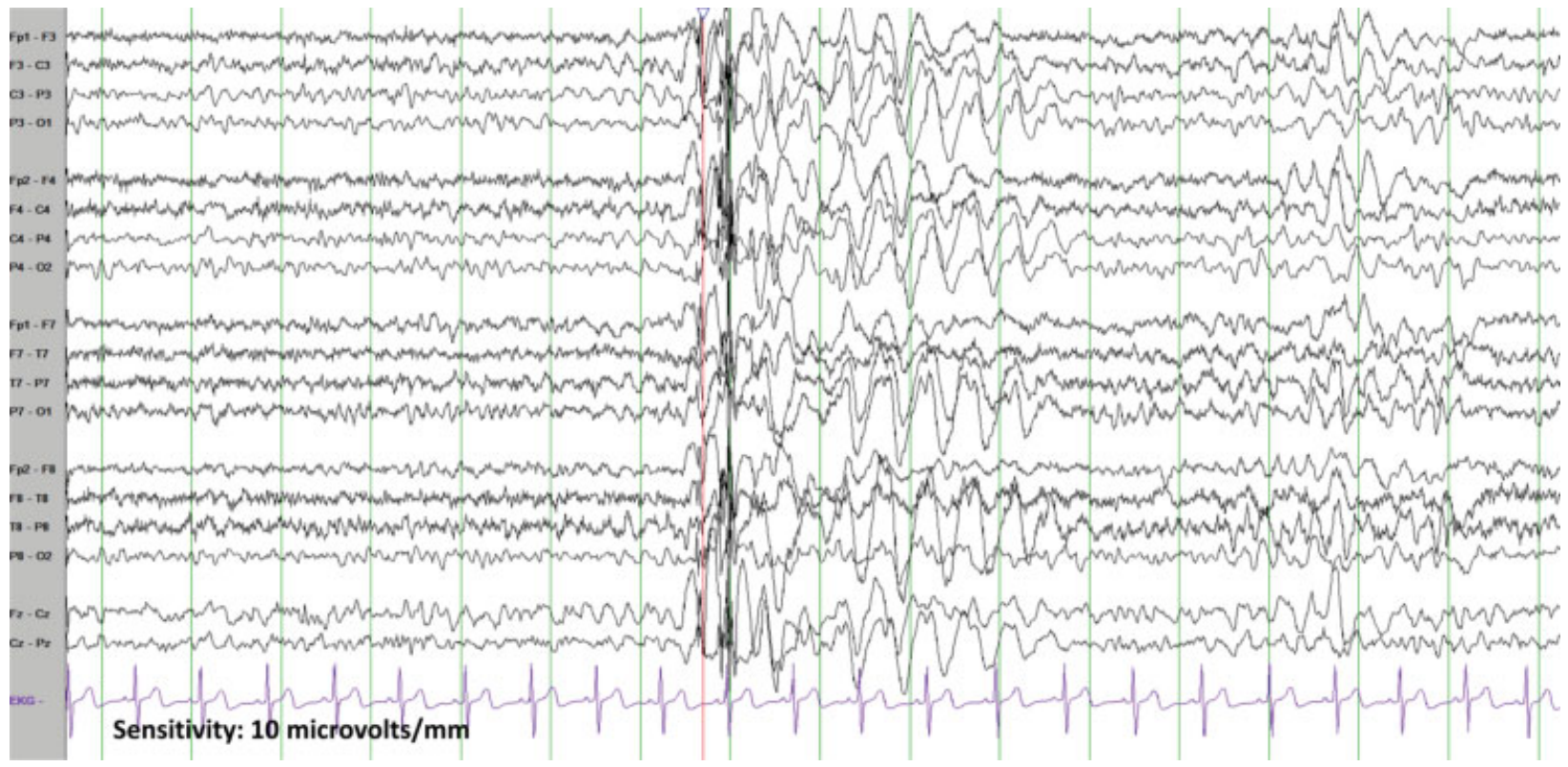

Fig. 4 Interictal EEG. Generalized medium- to high-amplitude spikes and polyspikes.

disorders, nonsyndromic epilepsy, and learning disabilities. ${ }^{9}$ In the seminal article to identify this gene in a large family with epilepsy and multiple seizure types, there was one patient with seizures only in the bath. ${ }^{10}$ Our patient has epilepsy and reflex bathing seizures, autism, and cognitive impairments.

SYN1 encodes a neuron-specific phosphoprotein implicated in the regulation of neurotransmitter release and synaptogenesis. ${ }^{9}$ Fassio et al evaluated SYN1 knockout mice and found that the defect appeared to be in synaptic vesicle trafficking as well as synaptic maturation and remodeling. In a mouse model, SYN1 expression was maximal at 3 to 4 weeks of life, which corresponds to a developmental period of extensive synaptic pruning. ${ }^{9}$ Our patient's SYN1 mutation

\section{SYN1 - Synapsin I, exon 10, NM_006950.3}

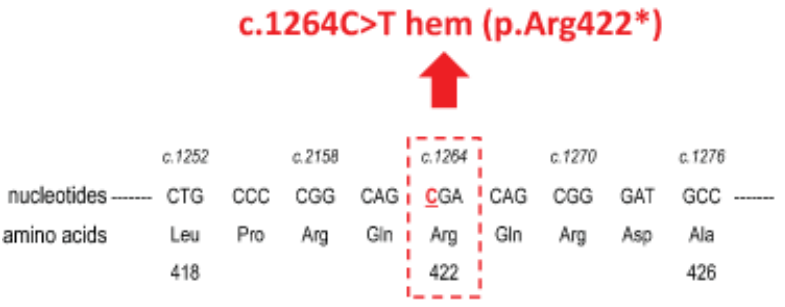

Fig. 5 SYN1 mutation identified from a 40 gene epilepsy panel. From the patient's genomic DNA, 40 genes were analyzed by both massively parallel sequencing and oligonucleotide array. Sequencing identified a single SYN1 variant at nucleotide position c.1264 in exon 10 (transcript reference NM_006950.3). This nucleotide change on the Xchromosome was hemizygous for this patient. As shown in the diagram, the nucleotide change from C to T at position c.1264 (red dashed box) results in a switch from an arginine (Arg) amino acid residue at position 422 to a stop codon that signals the premature termination of the amino acid translation. In comparison, wild type SYN1 is 705 amino acids in length. and clinical presentation add information to the literature about this rare genetic epilepsy and suggests that aberrant synaptic maturation could contribute to epileptogenic networks responsible for reflex epilepsy. His case also informs ongoing discussions surrounding the hypothesis that autism spectrum disorders occur due to abnormal synaptic function or neural connectivity when neuronal circuits are remodeled by experience. ${ }^{9}$

When making treatment decisions in bathing and reflex epilepsy, carbamazepine and oxcarbazepine have been reported to be superior to other ASMs. ${ }^{11}$ In our patient, no single medication was fully effective for reflex seizures. Of his various medication trials, carbamazepine was likely more helpful than other ASMs, though it was not able to adequately control his epilepsy.

Vagal nerve stimulation has been used to control reflex and nonreflex seizures. A case series describing three adult patients from Brazil described an improvement from baseline seizure frequency for both eating-related reflex seizures (70-95\% improvement), and nonreflex seizures (5-40\% improvement) in two of the three patients. ${ }^{12}$ It is theorized that peripheral input from organs innervated by the vagus nerve might be a part of the trigger-inducing eating-related seizures; therefore, stimulation of vagus nerve could help control the afferent input inducing these seizures. Our patient's response to VNS was largely confined to his spontaneous seizures. Further studies demonstrating the efficacy of VNS in reflex epilepsy and in particular bathing epilepsy are needed before drawing conclusions about the efficacy of VNS in these patients.

Stress is a commonly reported seizure trigger in people with epilepsy. Patients with uncontrolled epilepsy may have a heightened response to stress, increasing cortisol, and decreasing seizure threshold. ${ }^{13}$ Desensitization therapy has not been reported in the literature as a treatment 
modality for reflex epilepsies. Desensitization helps diminish fear and anxiety gradually. Our patient developed a fear of running water and bathing since he associated his seizures with these events. Desensitization therapy and family therapy by a child psychologist were utilized to help diminish his fear response, improve coping, and address family members' reactions to his seizures. His bathing-related seizures, rather than only his bathing-related anxiety, were improved with this therapy. It would be important to evaluate this therapy in other patients with reflex epilepsy and associated anxiety related to triggers.

In our patient's medically intractable epilepsy with reflex bathing seizures as well as spontaneous nocturnal seizures, the addition of nightly clonazepam and the placement of VNS reduced his nocturnal spontaneous seizures while the addition of desensitization therapy, when consistent, improved his reflex seizures.

This case adds knowledge regarding SNY1-associated reflex bathing seizures, epilepsy, and autism. This association has previously been reported in only one large FrenchCanadian family. ${ }^{8}$ Identification of this correlation may be helpful in recognition and treatment of other patients and families with this rare presentation. Identification of SYN1 mutation as the etiology for epilepsy may alert the clinician to increased risk for reflex bathing seizures and counseling about drowning risks in these patients. The role and spectrum of manifestations of SNY1 in reflex bathing seizures, epilepsy, and autism require further understanding, but testing for SYN1 mutation can be considered with in patients with reflex bathing seizures, epilepsy, and autism. Larger studies into efficacy of specific ASMs, VNS, and other epilepsy treatment modalities are needed to provide further insight into the optimal treatments for patients with this mutation.

\section{References}

1 Fisher RS, Acevedo C, Arzimanoglou A, et al. ILAE official report: a practical clinical definition of epilepsy. Epilepsia 2014;55(4): 475-482

2 Blume WT, Lüders HO, Mizrahi E, Tassinari C, van Emde Boas W, Engel J Jr. Glossary of descriptive terminology for ictal semiology: report of the ILAE task force on classification and terminology. Epilepsia 2001;42(9):1212-1218

3 Italiano D, Ferlazzo E, Gasparini S, et al. Generalized versus partial reflex seizures: a review. Seizure 2014;23(7):512-520

4 Xue LY, Ritaccio AL. Reflex seizures and reflex epilepsy. Am J Electroneurodiagn Technol 2006;46(1):39-48

5 Serrano-Castro PJ, Arjona A, Rubí-Callejón J, Alonso-Verdegay G, Huete-Hurtado A. [Advances in our knowledge of the causation and pathophysiology of reflex epilepsies] [in Spanish]. Rev Neurol 2006;43(12):745-752

6 Italiano D, Striano P, Russo E, et al. Genetics of reflex seizures and epilepsies in humans and animals. Epilepsy Res 2016;121:47-54

7 Meghana A, Sinha S, Sathyaprabha TN, Subbakrishna DK, Satishchandra P. Hot water epilepsy clinical profile and treatment-a prospective study. Epilepsy Res 2012;102(3):160-166

8 Nguyen DK, Rouleau I, Sénéchal G, et al. X-linked focal epilepsy with reflex bathing seizures: characterization of a distinct epileptic syndrome. Epilepsia 2015;56(7):1098-1108

9 Fassio A, Patry L, Congia S, et al. SYN1 loss-of-function mutations in autism and partial epilepsy cause impaired synaptic function. Hum Mol Genet 2011;20(12):2297-2307

10 Garcia CC, Blair HJ, Seager M, et al. Identification of a mutation in synapsin I, a synaptic vesicle protein, in a family with epilepsy. J Med Genet 2004;41(3):183-186

11 Feyissa AM, Patterson MC, Wong-Kisiel LC. Too old for a diaper! Child with diaper changing-induced seizures. Pediatr Neurol 2016;54:91-92

12 Cukiert A, Mariani PP, Burattini JA, et al. Vagus nerve stimulation might have a unique effect in reflex eating seizures. Epilepsia 2010;51(2):301-303

13 Allendorfer JB, Heyse H, Mendoza L, et al. Physiologic and cortical response to acute psychosocial stress in left temporal lobe epilepsy-a pilot cross-sectional fMRI study. Epilepsy Behav 2014;36:115-123 
